\title{
Xe interacting with porous silicon
}

\author{
Assaf Paldor, ${ }^{a}$ Gil Toker $^{a}{ }^{\text {Yigal Lilach }}{ }^{b}$ and Micha Asscher $* a$ \\ Received 17th December 2009, Accepted 29th March 2010 \\ First published as an Advance Article on the web 30th April 2010 \\ DOI: $10.1039 /$ b926692e
}

Thin films of porous silicon (PS), structurally characterized by HR-SEM, were studied using xenon Temperature Programmed Desorption (TPD) as a probe of its inner pores. Geometric hindrance of the depth desorbing population and multiple wall collisions result in a unique double-peak structure of the TPD curve. Surface-diffusion assisted adsorption mechanism into inner pores at $48 \mathrm{~K}$ is proposed as the origin of these unique TPD spectra. It is experimentally verified by mild $\mathrm{Ne}^{+}$sputtering prior to TPD which preferentially removes Xe population from the top surfaces. A pore-diameter limited desorption kinetic model that takes into account diffusion and pore depth well explains the governing parameters that determine the experimental observations. These results suggest that TPD may be employed as a highly sensitive, nondestructive surface area determination tool.

\section{Introduction}

Since the discovery of its luminescent properties in $1990,{ }^{1}$ the scientific and technological interest in porous silicon (PS) has been steadily increasing. Due to its unique properties PS is used in a wide variety of fields and applications, such as light emitting diodes, ${ }^{2}$ photonic crystals, ${ }^{3}$ biosensors ${ }^{4}$ and gas sensors. ${ }^{5}$

Many of these applications take advantage of PS's high surface area, which can be as much as $500 \mathrm{~m}^{2} \mathrm{~g}^{-1}$, depending on the fabrication conditions. The standard procedure for quantification and characterization of high surface area materials is the widespread $\mathrm{BET}^{7}$ method. However, it is limited in sensitivity, ${ }^{8-10}$ requiring samples having surface area of at least $1 \mathrm{~m}^{2}$. Temperature Programmed Desorption (TPD) of $\mathrm{N}_{2}$ has been used to characterize nanoporous films of metastable amorphous solid water (ASW). ${ }^{11,12}$ It was shown that new desorption peaks could be associated with the inner pores of ASW prepared specifically by molecular beam techniques in order to produce high porosity ASW films.

Here we report on the unique adsorption, desorption and diffusion processes of Xe on PS samples of limited surface area, estimated to be in the order of a few tens of $\mathrm{cm}^{2}$. The results of this study demonstrate the great potential of TPD as a highly sensitive surface area quantification method for porous materials.

\section{Experimental}

Rectangular samples, sized $18 \mathrm{~mm} \times 9 \mathrm{~mm}$, were cut from parent wafers (MaTeck) of $\mathrm{n}^{+}$type, (100) orientation and resistivity of $0.01-0.04 \Omega \mathrm{cm}$. Mesoporous samples of pore diameters $40 \pm 7 \mathrm{~nm}$ were fabricated in a single tank apparatus ${ }^{13}$ using the chronopotentiometry mode of an electrochemical

${ }^{a}$ Institute of Chemistry and the Farkas Center for Light Induced

Processes, The Hebrew University of Jerusalem, 91904, Israel.

E-mail:asscher@chem.ch.huji.ac.il

${ }^{b}$ Harvey Kruger Center for Nano-Fabrication,

The Hebrew University of Jerusalem, 91904, Israel workstation (CH-Instruments), a three-electrode setup (Si sample, $\mathrm{Ag}$ and $\mathrm{Pt}$ wires as the counter, reference and working electrodes, respectively) and an etching solution of (vol.) $1: 1$ : $2 \mathrm{HF}$ (Baker, aq. $49 \mathrm{wt} \%$.) : $\mathrm{H}_{2} \mathrm{O}$ : ethanol. An external DMM (Keithley) confirmed excellent current stabilization at $99 \mathrm{~mA}$, with good reproducibility between samples $(\sim 1 \mathrm{~mA})$. Upon removal of the sample from the etching solution it was squirted with isopropanol to allow drying in air in low-surface-tension environment, ${ }^{14}$ apparently important to avoid collapse of internal nano-pores during the solvent evaporation step.

Auger electron spectra (employing LK-Technologies instrument) of the freshly prepared PS samples indicated negligible oxidation during the exposure to ambient air ( $\sim 30 \mathrm{~min}$ ). K-type thermocouple wires were spot welded to a thin Ta foil, squeezed to the Si sample by one of the $\mathrm{W}$ clips that hold the sample against copper legs. The latter were attached via a sapphire disc to a closed-cycle He cryostat (APD). Sample temperature was typically $48 \pm 2 \mathrm{~K}$.

Xe (Matheson, 99.995\%) was introduced by backfilling the UHV chamber at pressures between $10^{-9}-10^{-7}$ Torr for maximal surface coverage uniformity. ${ }^{15}$ Xe exposures were monitored using a sensitivity-corrected nude Ion Gauge (Varian). A retractable quadrupole mass spectrometer (Extrel) was tuned to $m / z=64$, ascribed to doubly ionized ${ }^{128} \mathrm{Xe}$ isotope, for TPD measurements.

\section{Results and discussion}

Typical HR-SEM images of a top view and a cleaved cross section of the sample are shown in Fig. 1A and B, respectively. Fig. $1 \mathrm{C}$ reveals the existence of a bottleneck morphology: the pore diameter at depth is approximately twice the diameter at the pore's topmost $50 \mathrm{~nm}$ or so. The pore depth $(Z)$ is linearly dependent on the etching duration; under our typical etching conditions $(100 \mathrm{~mA})$ the etching rate was $120 \pm 4 \mathrm{~nm} \mathrm{~s}^{-1}$, as seen in Fig. 1D. 

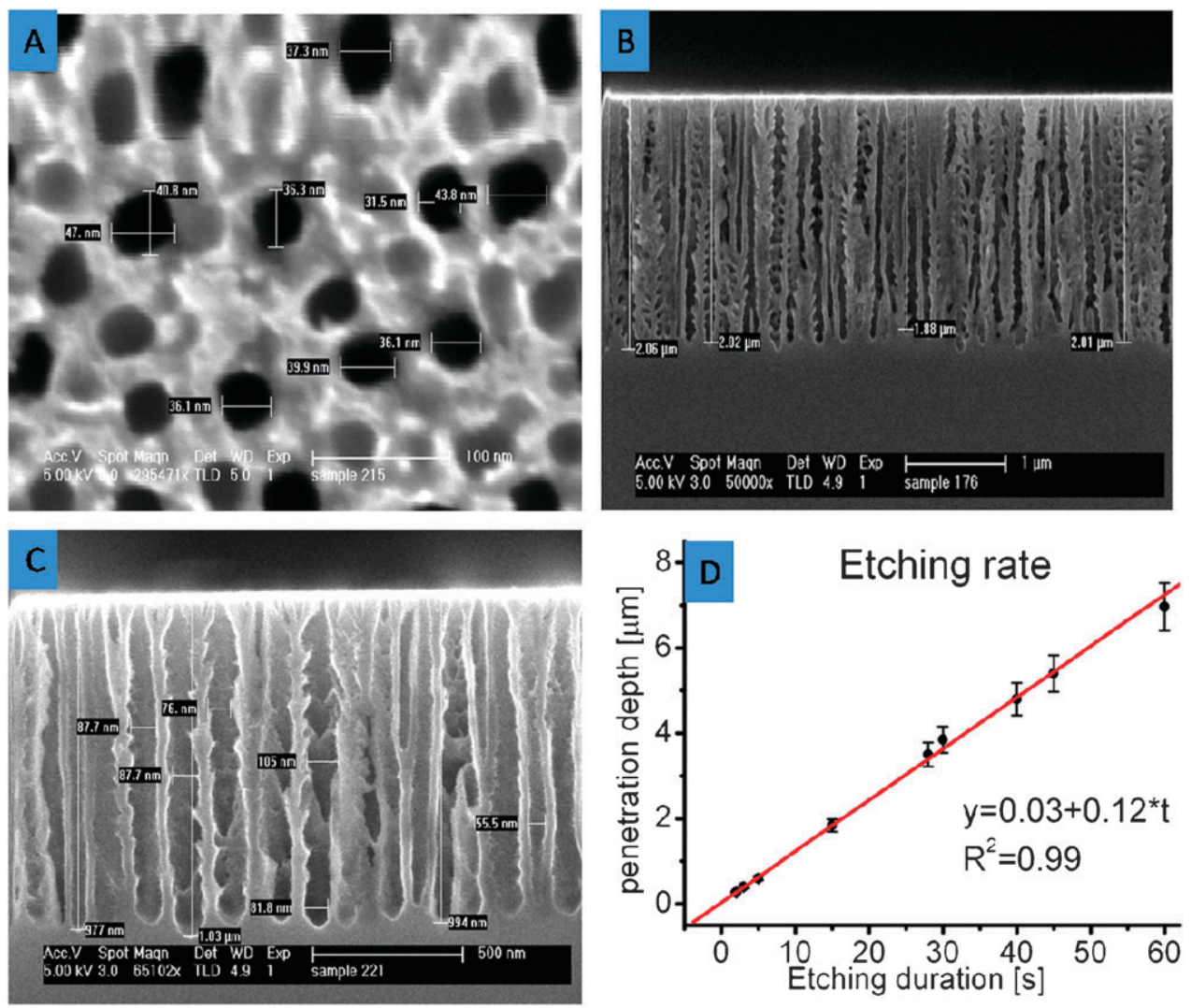

Fig. 1 (A) Top view and (B) cross section HR-SEM images of a PS sample. (C) Pores widen at depth resulting in a bottleneck morphology. (D) Linear dependence of the pore depth on the etching duration.

\subsection{TPD from flat $\mathrm{Si}-\mathrm{H}$ samples}

Desorption curves from reference silicon samples at a gradually increasing coverage (heating rate $\beta=5 \mathrm{~K} \mathrm{~s}^{-1}$ ) are shown in Fig. 2. These samples were prepared by immersion in the etching solution (no current applied) thus rendering them flat H-terminated crystalline $\mathrm{Si}(100)$ surfaces. ${ }^{16}$ TPD spectra of sub-monolayer coverage are shown as inset in Fig. 2.

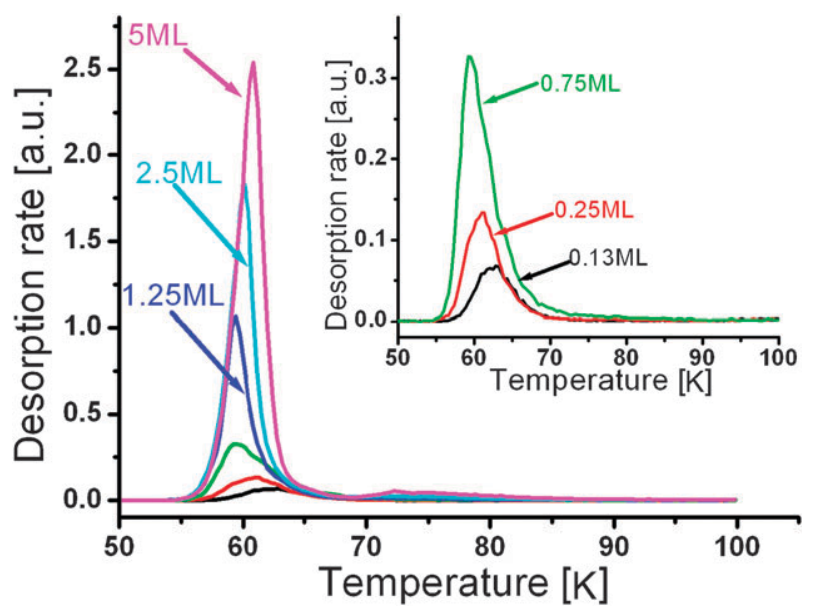

Fig. 2 TPD curves from a flat H-terminated sample exhibit a single narrow peak. The small peak at $T \approx 75 \mathrm{~K}$ represents desorption from surface defects or from the tungsten sample holders. Zoom in on sub-monolayer coverages is shown as inset.
Desorption from these samples has been concentrated in a single narrow peak near $60 \mathrm{~K}$ regardless of coverage. For high coverage an additional small Xe desorption peak emerges ( $\sim 1 \%$ of the primary peak) at higher temperatures. It is attributed to desorption either from surface defects where the binding is stronger or from the tungsten clips which lag after the sample's temperature ramp. The latter is more likely, as the former is expected to play a role at very low coverage as well.

At sub-monolayer coverage first order desorption kinetics is observed, with a shift of the peak desorption rate to lower temperature due to weak repulsive interactions between the adatoms. Further increase of the coverage alters the behavior towards zero order kinetics - the curves exhibit coalescence of their leading edges and the peak desorption rate shifts to higher temperature with increasing coverage. ${ }^{17,18}$ The gradual decrease in desorption kinetic order from first with lateral repulsions to zero is in accordance with many reports on desorption of Xe from metallic surfaces. ${ }^{19,20}$

A sticking coefficient of $0.5 \pm 0.1$ was deduced from adsorption curves. Assuming a pre-exponential factor of $10^{13}$ atoms $\mathrm{s}^{-1}$, the activation energy for desorption from the multilayer is $15 \pm 1.7 \mathrm{~kJ} \mathrm{~mol}^{-1}$. This value is in agreement with literary values of the binding energy of Xe multilayers on metallic surfaces, thus stressing the insensitivity of the Xe multilayer binding energy to the substrate. In the multilayer regime, the sticking coefficient gradually decreases to approx. $0.2 \pm 0.1$. 


\subsection{TPD from porous silicon}

In contrast to the flat $\mathrm{Si}-\mathrm{H}$ surface, Fig. $3 \mathrm{~A}$ reveals the same experiments conducted on a $3.5 \mu \mathrm{m}$ deep porous sample with an average pore diameter of $40 \mathrm{~nm}$. The single narrow desorption peak from the flat sample clearly spreads into two distinct peaks when the sample is rendered porous.

Lacking crystalline order, ascription of the adatom density in monolayer coverage is impossible, and consequently so is the deduction of the sticking coefficient. However, for clarity and consistency with the flat reference samples the same ML yardstick was used, although it surely does not represent the true surface coverage of the porous samples.

Fig. 3B compares the desorption curves from the two samples (flat and porous, pore depth $=3.5 \mu \mathrm{m}$ ) for a representative Xe coverage of $2.5 \mathrm{ML}$. The onset temperature for desorption ( $T_{\text {onset }}$ ) from the flat sample is approximately $5 \mathrm{~K}$ lower than for desorption from the porous sample. High density of defects of various kinds on the flat, corrugated areas of the porous samples may explain this shift.

Nevertheless, aside from differences in $T_{\text {onset }}$ and a dramatic reduction in amplitude, the former of the two peaks resembles the flat sample's single peak in its shape, width and trends with increasing coverage. The latter peak, in contrast, is much wider, appears at elevated temperatures, and the leading edges of its desorption curves do not seem to coalesce. These
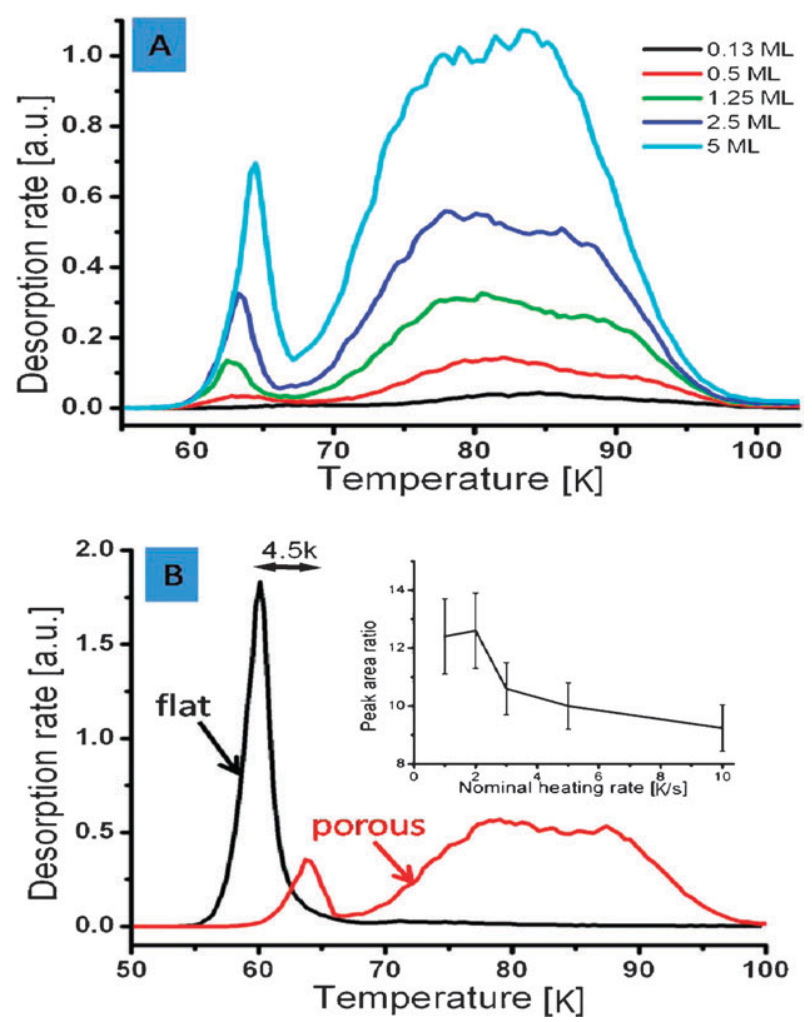

Fig. 3 (A) TPD from a porous sample, $Z=3.5 \mu \mathrm{m}, \beta=5 \mathrm{~K} \mathrm{~s}^{-1}$. (B) A representative $2.5 \mathrm{ML}$ experiment from both the flat $\mathrm{Si}-\mathrm{H}$ and the porous samples. Inset: variation of the ratio between the areas under the two peaks (2nd to 1st, namely higher temperature to lower temperature) as a function of the nominal heating rate for a $6 \mu \mathrm{m}$ deep sample. observations lead to the conclusion that the first of the two peaks represents desorption from the top surface (and perhaps from the topmost part of the pore), whereas the second peak represents desorption from pore depth. Even on a highly corrugated surface, a wide distribution of the binding energy cannot solely account for such a large discrepancy in the desorption temperature, particularly in the multilayer regime where the interactions are predominantly Xe-Xe.

To further stress this argument we have etched a sample for $100 \mathrm{~ms}$ only. SEM characterization verified that such a short etching session renders the surface highly corrugated much like the porous samples, yet does not form pores. The desorption curves from this sample exhibited a single desorption peak slightly wider and at a higher temperature than the flat $\mathrm{H}$-terminated reference sample, but by no means similar to the double peak structure observed for the porous samples. Therefore, it can be concluded that the appearance of the second peak at elevated temperatures where the desorption from the top surface is long gone, is due to the journey the atoms desorbing at pore depth must undergo, thus delaying their motion towards the detector. Namely, their detection at higher temperatures is not because they desorbed later, but rather due to the occurrence of multiple desorption-readsorption cycles on the inner pore walls, as a result of which their detection is delayed to higher temperature.

This understanding is also implied by the different effect of $\beta$, the nominal heating rate, on the two peaks, as shown in the inset of Fig. 3B for a $6 \mu \mathrm{m}$ deep sample: the ratio between the areas of the two peaks (2nd to 1st) decreases with increasing heating rate. This attests the existence of an extraneous (namely, other than simple desorption) rate limiting process which applies to the latter desorbing population but not to the former, i.e. the need to get out of the pores. This trend has been observed for samples with shallower pores as well, although not as pronounced, indicating that the fraction of atoms to which this step applies increases with increasing pore depth, as expected.

Several studies have reported ${ }^{21,22}$ on the desorption kinetics of Xe from bundles of single wall carbon nanotubes (SWCNTs). These structures resemble PS in some aspects, but unlike our system in which desorption from two essentially different surfaces forms the double peak structure, Xe adsorbs only on the external bundle surfaces of the SWCNT; its access to the endohedral sites is blocked, and thus the SWCNTs exhibit only a single desorption peak. Aside from this fundamental difference, other findings are analogous: the single desorption peak from SWCNT was significantly broader with respect to the HOPG reference surface and shifted to higher temperatures, much like the high temperature peak in the desorption curve observed for PS. These authors have concluded that diffusion of the desorbing particles through the porous structure of the SWCNTs dominates the desorption kinetics.

\subsection{Pulsed heating}

In order to further investigate the effect of heating rate at values above the standard rates, we have introduced a pulsed heating procedure. 
An abrupt current discharge from a $32 \mu \mathrm{F}$ capacitor through the silicon sample was used to induce transient heating of the sample from $48 \mathrm{~K}$ to $58 \pm 1 \mathrm{~K}$. The typical rise time of the current pulse was $\sim 0.15 \mathrm{~s}$, the width at half maximum is $0.17 \pm 0.03 \mathrm{~s}$ and it takes about $0.5 \mathrm{~s}$ for the sample to cool back to its baseline temperature. Typically, $20 \mathrm{~s}$ were idly waited between consecutive pulses. The pulses were applied following adsorption (initial coverage $2.5 \mathrm{ML}$ ) and prior to desorption $\left(\beta=5 \mathrm{~K} \mathrm{~s}^{-1}\right)$, so that the differences between a standard and a post-pulse TPD curve would demonstrate the effect of pulsed heating on the Xe adatom population.

The area under the curves in Fig. 4A reveals that $90 \%$ of the adatom population on the flat surface was eliminated by the first pulse. Apparently, the rapid re-cooling precluded dissipation of heat to the tungsten clips, thus giving rise to traces of desorption ( $4 \%$ of the original area) after 100 pulses, as seen in the inset therein. In contrast, almost $40 \%$ of the atoms adsorbed on the porous sample $(Z=3.5 \mu \mathrm{m})$ survived 1000 pulses, as seen in Fig. 4B. Particularly, while the first peak was
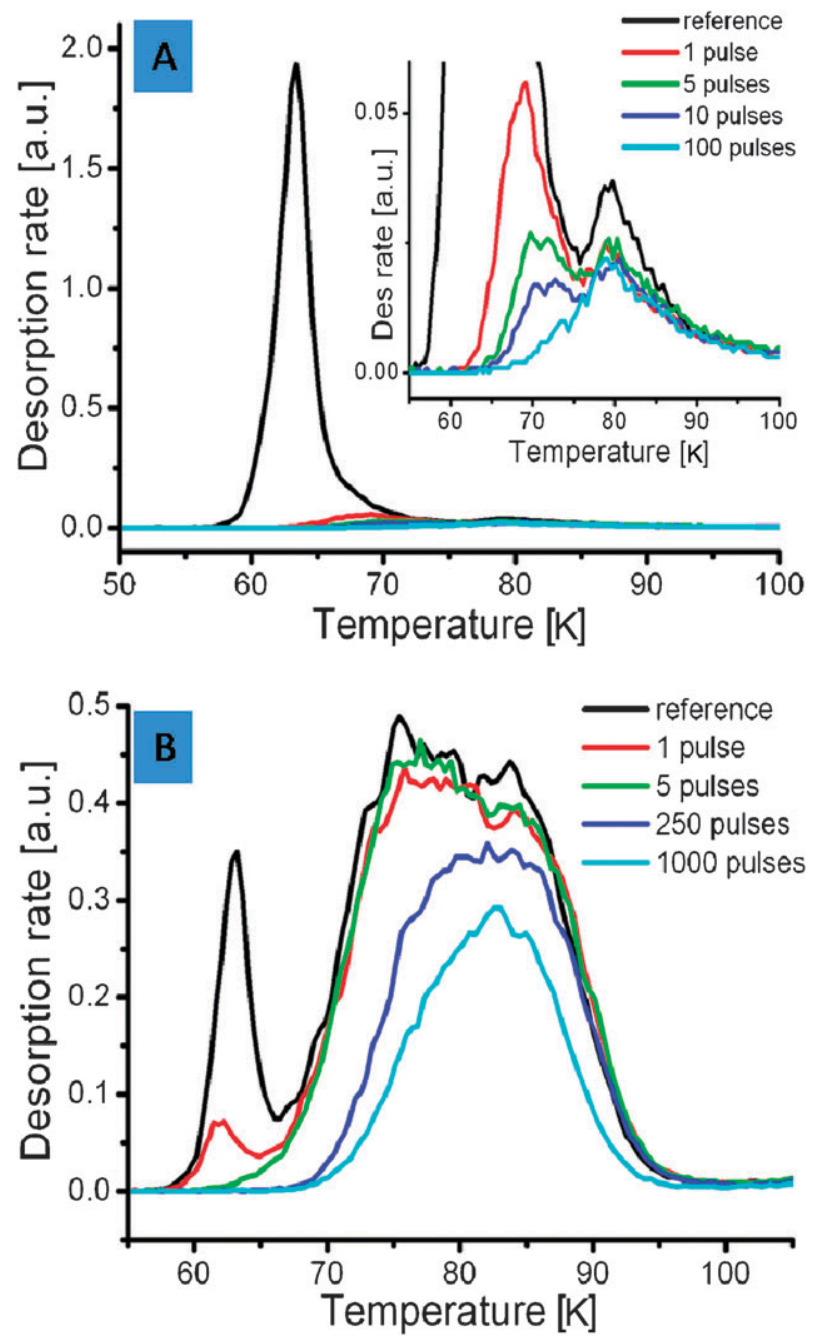

Fig. 4 Effect of abrupt heating pulses on the desorption curve from (A) a flat sample and (B) a porous sample, $Z=3.5 \mu \mathrm{m}$. Initial coverage $2.5 \mathrm{ML}, \beta=5 \mathrm{~K} \mathrm{~s}^{-1}$. readily diminished by the pulses much like the flat sample's single peak, the adatoms in the deep porous domains are barely affected by these pulses.

The pore-desorption interpretation of these findings is straightforward: the $0.5 \mathrm{~s}$ interval before re-cooling was enough for the atoms desorbing from the top surface to be pumped away. In contrast, for the atoms desorbing from the bottom of the pore the $0.5 \mathrm{~s}$ was too short a period to get out of the pore, and thus they were re-adsorbed at pore depth again, waiting for the next pulse.

It should be noted that for the porous samples, idle (no pulsed heating) waiting periods of durations comparable to the longer series of pulses resulted in considerable spontaneous decay of the overall Xe coverage, indicating the occurrence of isothermal desorption. However, the selective depletion of the first pulse is doubtlessly due to the heating pulses and not to isothermal desorption, since the overall double-peak structure, the relative amplitudes of the two peaks and the width of the curve persisted during the isothermal depletion process.

After a few hundreds of pulses the desorption onset gradually shifts to higher temperature. This is not surprising, since another look at Fig. 3A reveals that even in standard TPDs the onset temperature for the second peak increases with decreasing coverage. Each pulse makes a double contribution to this upward temperature shift: (a) In addition to the depletion of the adatom population inside the pores, each pulse also induces transient desorption which homogenizes the coverage along the pore walls. Therefore, each pulse slightly reduces the internal adatom density, and due to the repulsive interactions slightly increases $T_{\text {onset }}$ of the subsequent desorption curve. (b) If there is a distribution of binding energies of $\mathrm{Xe}$ to $\mathrm{Si}-\mathrm{H}$ sites on the highly corrugated pore wall, the pulses selectively deplete the weaker binding sites.

\subsection{Role of pore depth}

The influence of pore depth on the second, high temperature Xe desorption peak is shown in Fig. 5, without changing any of the other morphological parameters. For Xe coverage of 2.5 ML, the results show that while the first desorption peak

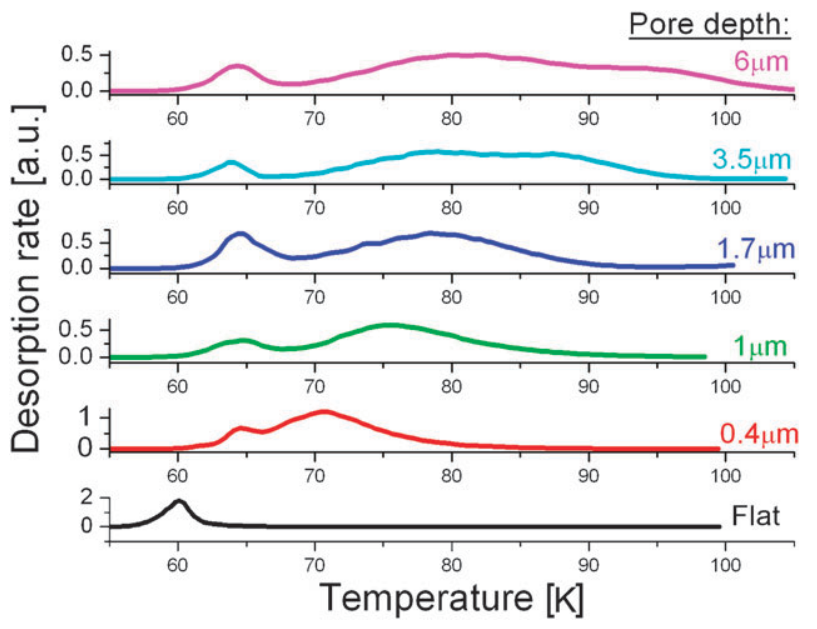

Fig. 5 The effect of variations in pore depth (indicated in micrometres) on the desorption curve in a $2.5 \mathrm{ML} \beta=5 \mathrm{~K} \mathrm{~s}^{-1}$ experiment. 
and the separating dip are barely affected by this change, deepening the pores further widens the second peak. This reinforces the pore-desorption notion, since when the pores are deepened the very last detected atoms require longer times to reach the detector, thus pushing the trailing edge of the curve further towards higher temperatures.

\subsection{Kinetic model: pore geometry limited desorption}

So far we have established the understanding that the atoms desorbing at pore depth are subject to an additional rate limiting step other than the desorption kinetics. To better understand this step both diffusion and simple geometric effects of the pore shape need to be considered. In the following section we describe a kinetic model which attempts to evaluate the importance of these effects. The mean free path within the pores is comparable with the pore diameter and thus the collisions with the pore walls play a dominant role. In such multiple encounters, Xe atoms desorb then re-adsorb and spend a characteristic temperature-dependent residence time on the pore wall surfaces before desorbing again and eventually proceeding out of the pores towards the detector. The residence time can be estimated using the inverse of the expression for (first order) desorption rate constant:

$$
k_{\mathrm{des}}=\nu_{\mathrm{D}} \exp \left(-E_{\mathrm{des}} / k_{\mathrm{B}} T\right)
$$

where $k_{\text {des }}$ is the desorption rate constant, $E_{\text {des }}$ is the activation energy for desorption, $\nu_{\mathrm{D}}$ is the pre-exponential factor, $T$ is the surface temperature and $k_{\mathrm{B}}$ is Boltzmann constant.

Assuming typical values of $E_{\mathrm{des}}=15.9 \mathrm{~kJ} \mathrm{~mol}^{-1}$ and $\nu_{\mathrm{D}}=10^{13} \mathrm{~s}^{-1}$, we obtain a residence time of $7 \mathrm{~s}$ at a surface temperature of $60 \mathrm{~K}, 70 \mathrm{~ms}$ at $70 \mathrm{~K}$ and $2.5 \mathrm{~ms}$ at $80 \mathrm{~K}$. This implies that during a desorption experiment all the atoms in the pore can be considered as adsorbed on its walls. Any gas phase effects, such as diffusion or collisions within the open volume of the pores, are negligible.

Using these arguments, we have simulated the desorption process of the atoms out of the pores coupled to diffusion on the pore walls. As schematically shown in Fig. 6, a model cylindrical pore was divided into thin sections of thickness $\mathrm{d} z$, the outer rims of which are the pore walls. Atoms desorbing from each section will manage to escape out of the pore only

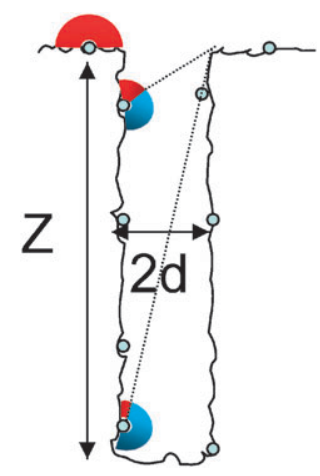

Fig. 6 A scheme of the pore-desorption as suggested in the simulated kinetic model. if they desorb in the direction of the pore opening. The probability that this will happen from a section in depth $Z$ is the solid angle of the top pore hole as seen from this depth. This value is given by $B=d^{2} / 2\left(Z^{2}+d^{2}\right)$ with $d$ the hole radius. The total desorption rate from each section is then given by: $k_{\mathrm{des}} \theta^{n} B$ ( $n$ is the desorption order), where $B=1$ for sections outside the pore, on the top surface. The atoms that did not escape are assumed to be re-adsorbed in the same depth $Z$. This is a significant simplification of the situation, but desorption and readsorption from neighboring sections should almost balance out. The simulated TPD signal is proportional to the decrease in total coverage in a given time interval $\mathrm{d} t$.

Another possibility for atom movement outside the pore is by surface diffusion on the pore walls. An activated diffusion process is introduced:

$$
D(T)=D_{0} \exp \left(-E_{\mathrm{diff}} / k_{\mathrm{B}} T\right)
$$

where $E_{\text {diff }}$ is a coverage independent activation energy for wall-diffusion of adatoms and $D_{0}$ is the corresponding pre-exponential factor.

Assuming that $D$ is independent of $z$, the spatial coordinate along the pore axis (perpendicular to the surface), Fick's second law of diffusion becomes:

$$
\partial C / \partial t=D(T) \partial^{2} C / \partial z^{2}=D_{0} \exp \left(-E_{\mathrm{diff}} / k_{\mathrm{B}} T\right) \partial^{2} C / \partial z^{2}
$$

where $c$ is the surface coverage at section $Z$.

Initial uniform adatom coverage along the pore walls (namely, $c(z)=$ constant) and a constant binding energy are assumed. The desorption pre-exponent was fixed at $10^{13} \mathrm{~s}^{-1}$ and the Xe activation energy for desorption was $15.5 \mathrm{~kJ} \mathrm{~mol}^{-1}$. The activation energy for diffusion was set to $7.5 \mathrm{~kJ} \mathrm{~mol}^{-1}$, and $D_{0}$ to $10 \mathrm{~cm}^{2} \mathrm{~s}^{-1}$, only negligibly affecting the Xe desorption curves.

This model qualitatively explains the experimental data, particularly for thin porous layers.

The results of the kinetic model are shown in Fig. 7, revealing the effects of coverage and pore depth on the shape of the TPD curves. Fig. 7A shows that increasing the coverage

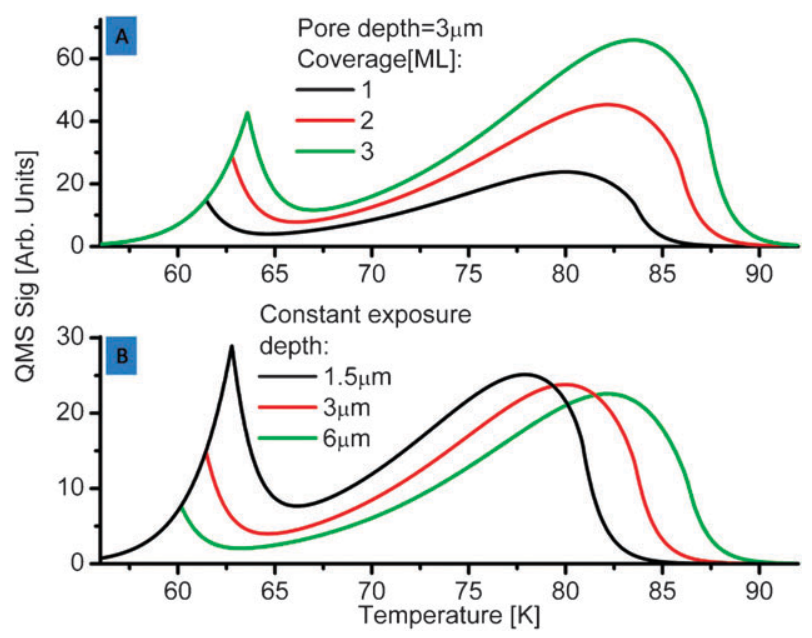

Fig. 7 Simulated TPD based on the above kinetic model showing the effect of (A) Xe coverage and (B) pore depth. 
gradually shifts both TPD peaks to higher temperature. The first Xe TPD peak behaves as expected from typical zero order desorption kinetics. The second broad peak is attributed to desorption from pore-depth. Multiple desorption-readsorption cycles result in a wider peak that shifts to higher temperature as coverage increases. Both peaks in Fig. 7A qualitatively reconstruct the experimental observations shown in Fig. 3.

Fig. 7B shows simulated TPD curves differing in pore depth. In the simulation, this inherently means a decrease in the effective density of the $\mathrm{Xe}$ adatoms, as the total number of $\mathrm{Xe}$ atoms remains fixed while the pores deepen. Consequently, the first peak which represents desorption from the top surface shifts to lower temperature, in accordance with its experimental behavior in Fig. 3A when the coverage decreases. More importantly, the second peak in Fig. 7B exhibits widening and shifting towards higher temperature when the pores are deepened, thus reconstructing the experimental observations shown in Fig. 5.

A demonstration of the relevance of this simulation to other gases is shown in Fig. 8. Two TPD experiments performed using $\mathrm{Xe}$ and $\mathrm{N}_{2} \mathrm{O}$ on the same porous sample (1.7 $\mu \mathrm{m}$ deep) are displayed. The two simulations presented (dashed red lines) differ only in gas parameters (mainly the desorption activation energy and diffusion parameters) while the parameters describing the pore (diameter, depth) were held fixed.

Lack of complete quantitative agreement between the experiments and the simulated TPD spectra is primarily attributed to a relatively wide distribution of activation energies for desorption. This is expected within the pores, as they are highly non-uniform in nature. Better agreement with the experimental data can be achieved for deeper samples when assuming two different populations (one distinct activation

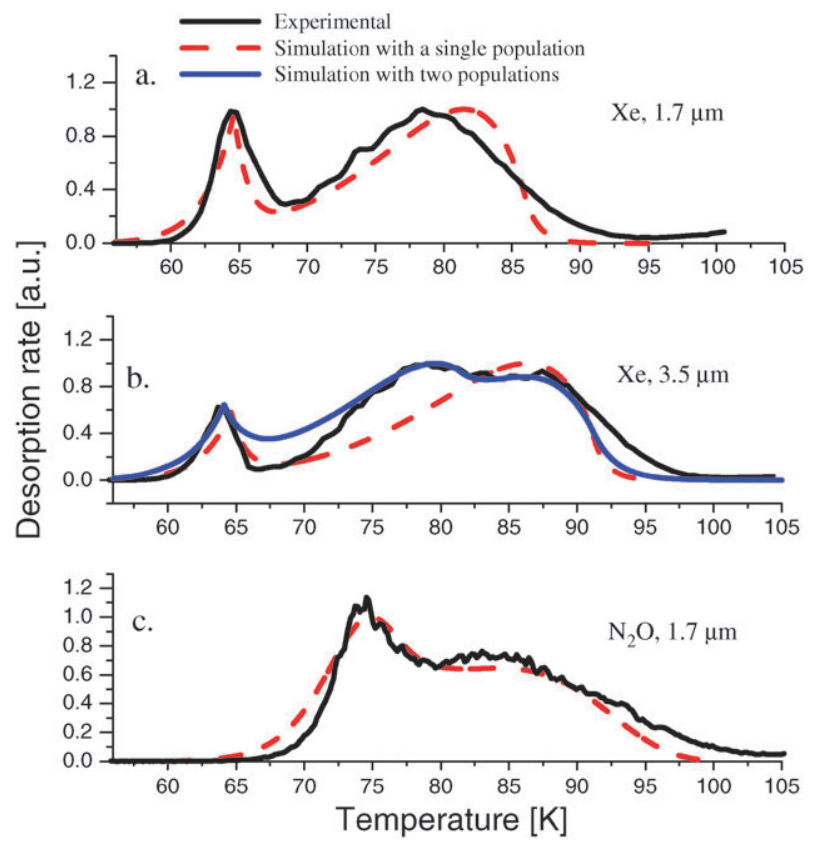

Fig. 8 A comparison between the simulation (dashed red line) and the experimental (solid black line) results. a. Xe on $1.7 \mu \mathrm{m}$ pores, b. Xe on $3.5 \mu \mathrm{m}$ pores and c. $\mathrm{N}_{2} \mathrm{O}$ on $1.7 \mu \mathrm{m}$. energy for desorption of each population) inside the pores, as can be seen in Fig. 8b (solid blue line).

Nevertheless, the qualitative resemblance between the experimental and simulated curves indicates that the geometrical inhibition and the activated diffusion play a dominant role in the desorption kinetics of Xe from the internal porous domain and are responsible for the resulting double peak structure of the TPD curves.

\subsection{Adsorption onto porous silicon}

We now consider the adsorption process. Based on top view SEM images (see Fig. 1), the pores constitute $\sim 10 \%$ of the top surface. Therefore, the probability for an impinging atom to directly strike the pore rather than a top-surface adsorption site is about $10 \%$. Consequently, the reduction in the area of the low temperature peak which represents desorption from the top surface is expected to be of similar magnitude. However, the reduction is far more dramatic, as seen in Fig. 3B above. This indicates that to a certain extent the internal pore sites replace those of the top surface, rather than add on to them. Similarly, the aforementioned $\sim 5 \mathrm{~K}$ increase in $T_{\text {onset }}$ (Fig. 3A above) further suggests that the adatom density on the top surface of the porous samples is smaller than the adatom density on the surface of the flat sample. Low density $\mathrm{Xe}$ adatoms primarily sample the corrugated sites which are of somewhat higher binding energy, and do not affect neighbor atoms via repulsive interactions, as occurs in high coverage.

Additionally, as $\mathrm{Xe}$ is backfilled into the chamber, the angular distribution of the impinging atoms is isotropic. Therefore, the chances of an approaching atom to "fly" all the way down to the bottom of the pore without adsorbing to the walls at higher regions of the pore are very small. This limitation is worsened by the existence of the bottleneck morphology (Fig. 1), which causes only a small fraction of the internal surfaces to have a direct line of sight of the impinging flux.

The emerging conclusion is as follows: direct adsorption from the gas phase occurs almost exclusively on the accessible domains, namely the (corrugated) top surface and the topmost parts of the pore. Following adsorption to these accessible domains the Xe atoms are diffusionally sucked into the pores driven by strong surface coverage gradients. Assuming a small ( $\sim 0.3-0.4)$ ratio $\Omega=E_{\mathrm{diff}} / E_{\mathrm{des}}$, allows for rapid diffusion of the highly mobile adatoms at temperatures well below the onset temperature for desorption $\left(T_{\text {onset }}\right)$. The barrier for this motion of the adatoms cannot be directly measured but an indirect indication arises from the variation of the sticking coefficient, as we describe below.

Due to multiple reflections, the sticking coefficient inside the pores may be assumed to be unity. Consequently, the weighted sticking coefficient for the porous sample in the submonolayer regime should be $0.5 \times 0.9+0.1 \times 1=0.55$, as the pores constitute only $10 \%$ of the top surface. Similarly, in the multilayer regime where the flat surface's sticking coefficient is reduced to 0.2 , the same calculation for the porous samples predicts a weighted sticking coefficient of $0.2 \times 0.9+0.1 \times$ $1=0.28$. In practice, however, the 0.4 and $6 \mu \mathrm{m}$ deep samples 


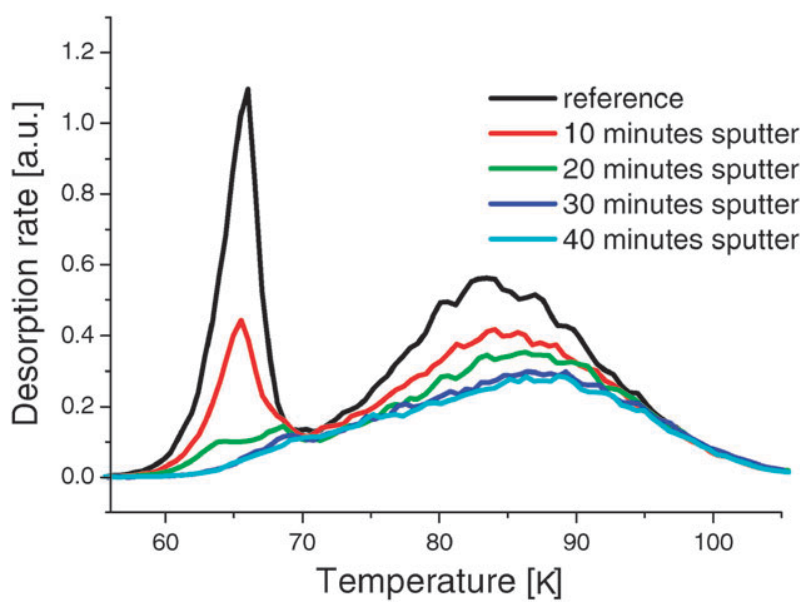

Fig. 9 Desorption spectra of $5 \mathrm{ML} \mathrm{Xe}$ from a porous sample $\left(Z=2 \mu \mathrm{m}, \beta=5 \mathrm{~K} \mathrm{~s}^{-1}\right)$ following $\mathrm{Ne}^{+}$sputter (ion energy = $200 \mathrm{eV}$, ion current $=1 \mu \mathrm{A}$ ) for different exposure times.

exhibited a 2-fold and 3.5-fold increase in their Xe uptake, respectively, with respect to the flat sample. This means that the existence of the pores does more than simply render $10 \%$ of the surface highly adsorptive; it significantly enhances the adsorption on the parts of the surface where pores were not formed. This implies that the presence of the pores slows down the completion of the monolayer on the top surface and the consequent reduction in the sticking coefficient, thus attesting constant evacuation of the adatoms from the top surface into internal pore domains. Particularly, when the pores are deeper, more atoms can be sucked away from the top surface resulting in the higher (sub-monolayer) apparent sticking coefficient and increased Xe uptake.

This hypothesis of adsorption mechanism implies that significant lateral diffusion of the $\mathrm{Xe}$ adatoms takes place during exposure at sample temperature of $48 \mathrm{~K}$. This conclusion can be rationalized if we assume the diffusion barrier to be no more than $7.5 \mathrm{~kJ} \mathrm{~mol}^{-1}$ (50\% of the barrier for desorption) which leads to a corresponding diffusion hopping time of less than $1 \mu$ s, calculated at the exposure temperature (48 K).

Further supporting evidence for the role of Xe surface diffusion at the adsorption temperature can be seen in Fig. 9. A porous sample $(Z=2 \mu \mathrm{m})$ was sputtered by medium energy $(200 \mathrm{eV}) \mathrm{Ne}^{+}$ions following exposure to $5 \mathrm{ML}$ Xe at the adsorption temperature of $48 \mathrm{~K}$. The resulting TPD spectra clearly reveal that 20 minutes of sputter time are sufficient to remove the low temperature peak. In contrast, the wider and high temperature peak attributed to desorption from pore-depth, cannot be eliminated and is practically fixed beyond 30 minutes sputter time at the same ion energy. The $\mathrm{Ne}^{+}$ions cannot sputter-remove the Xe atoms adsorbed at the inner pores. This observation indicates that $\mathrm{Xe}$ atoms do populate the inner pores already at the adsorption temperature via surface mobility mechanism discussed above. The fact that about half of the pore-depth desorption peak vanishes as a result of sputter, however, cannot rule out the possibility that at the adsorption temperature a larger fraction of the $\mathrm{Xe}$ atoms reside on the top surface and subsequently find their way to the inner pores (without sputter) while heating the surface during the TPD experiment.

\section{Conclusions}

TPD of Xe from porous silicon was demonstrated to be a sensitive way to probe pore morphology (depth) and indirectly its inner surface area. It was shown that desorption from inner pores results in a separate, wide TPD peak that emerges at gradually higher temperatures as the pores become deeper. Our results suggest that upon adsorption at $48 \mathrm{~K}$, Xe atoms migrate on the top surface and subsequently are sucked into the (bare) pores, this way populating the inner walls. A kinetic desorption model that accounts for the pore's diameter and depth has been constructed. This model reveals that the origin of the separate, high temperature and wide TPD peak is the delayed desorption due to the coupled multiple wall collisions-desorption events that take place within the inner pores.

It is speculated that inner surface areas two to three orders of magnitude smaller than the lower limit for commercially available techniques for surface area determination such as BET could be detected based on TPD measurements of the type described here.

Moreover, it is expected that similar methods of TPD based analysis can be applied to a wide variety of porous materials, which are becoming exceedingly important for nanotechnology.

\section{Acknowledgements}

Partial support by the Israel Science Foundation is acknowledged. The support of the technical staff of the Hebrew University Harvey Kruger Center for Nano Characterization (UNC) and Nano Fabrication (UNF) is acknowledged.

\section{References}

1 L. T. Canham, Appl. Phys. Lett., 1990, 57, 1046.

2 P. Steiner, F. Kozlowski and W. Lang, Appl. Phys. Lett., 1993, 62, 2700.

3 T. V. Murzina, F. Yu. Sychev, E. M. Kim, E. I. Rau, S. S. Obydena, O. A. Aktsipetrov, M. A. Bader and G. Marowsky, J. Appl. Phys., 2005, 98, 123702.

4 L. De Stefano, L. Rotiroti, I. Rendina, L. Moretti, V. Scognamiglio, M. Rossi and S. D'Auria, Biosens. Bioelectron., 2006, 21, 1664-1667.

5 J. M. Lauerhass, G. M. Credo, J. Heinrich and M. J. Sailor, J. Am. Chem. Soc., 1992, 114, 1911.

6 D. Kovalev, V. Yu. Timoshenko, N. Kunzner, E. Gross and F. Koch, Phys. Rev. Lett., 2001, 87, 068301.

7 S. Brunauer, P. H. Emmet and E. Teller, J. Am. Chem. Soc., 1938, 60, 309A.

8 J. Salonen, M. Bjorkvist and E. Laine, J. Appl. Crystallogr., 2000, 33, 504.

9 H. Yanazawa, K. Oshika and T. Matsuzawa, Adsorption, 2000, 6, 73-77.

10 I. Suzuki and K. Oosawa, Rev. Sci. Instrum., 1997, 68, 12.

11 G. A. Kimmel, K. P. Stevenson, Z. Dohnalek, R. S. Smith and B. D. Kay, J. Chem. Phys., 2001, 114, 12.

12 T. Zubkov, R. S. Smith, T. R. Engstrom and B. D. Kay, J. Chem. Phys., 2007, 124, 184708.

13 A. Halimaoui, Porous silicon science and technology, ed. J. C. Vial and J. Derrien, Springer-Verlag, Berlin, Heidelberg, 1994.

14 A. G. Cullis, L. T. Canham and P. D. J. Calcott, J. Appl. Phys., 1997, 82, 909.

15 G. Kerner, O. Stein, Y. Lilach and M. Asscher, Phys. Rev. B: Condens. Matter, 2005, 71, 205414. 
16 H. Ubara, T. Imura and A. Hiraki, Solid State Commun., 1984, 50, 673-675.

17 J. A. Venables and N. Bienfait, Surf. Sci., 1976, 61, 667.

18 H. Asada and M. Masuda, Surf. Sci., 1989, 207, 517.

19 B. Lehner, M. Hohage and P. Zeppenfeld, Chem. Phys. Lett., 2003, 369, 275-280.
20 W. Widdra, P. Trischberger, W. Frieb, D. Menzel, S. H. Payne and H. J. Kreuzer, Phys. Rev. B: Condens. Matter, 1998, 57, 7.

21 H. Ulbricht, J. Kriebel, M. Gunar and T. Hertel, Chem. Phys. Lett., 2002, 363, 252.

22 A. Kuznetsova, J. T. Yates Jr, J. Liu and R. E. Smalley, J. Chem. Phys., 2000, 112, 21. 